\title{
A biphasic response of polymerized Type 1 collagen architectures to dermatan sulfate
}

\author{
Konkada Manattayil Jyothsna ${ }^{1} \quad$ | Purba Sarkar ${ }^{2}$ ～Keshav Kumar Jha ${ }^{1,3}$ | \\ Lal Krishna A. S. ${ }^{1}$ | Varun Raghunathan ${ }^{1}$ | Ramray Bhat ${ }^{2}{ }^{\circ}$
}

${ }^{1}$ Department of Electrical Communication Engineering, Indian Institute of Science,

Bangalore, Karnataka, India

${ }^{2}$ Department of Molecular Reproduction, Development and Genetics, Indian Institute of Science, Bangalore, Karnataka, India

${ }^{3}$ Department of Functional Interfaces, Leibniz Institute of Photonic Technology, Jena, Germany

\section{Correspondence}

Varun Raghunathan and Ramray Bhat, Department of Electrical Communication Engineering, Indian Institute of Science, Bangalore, Karnataka 560012, India.

Email: ramray@iisc.ac.in (R. B.) and

Email: varunr@iisc.ac.in (V.R.)

Funding information

Department of Biotechnology, Ministry of Science and Technology, India, Grant/Award Number: BT/PR26526/GET/119/92/2017; Department of Science and Technology, India, Grant/Award Number: INT/Korea/P-44; Indian Institute of Science, Grant/Award Number: IE/ CARE-19-0319; Science and Engineering Research Board, Grant/Award Number: ECR/2015/000280; The Wellcome Trust DBT India Alliance, Grant/Award Number: IA/ I/17/2/ 503312

\begin{abstract}
Collagen I, the most abundant extracellular matrix (ECM) protein in vertebrate tissues provides mechanical durability to tissue microenvironments and regulates cell function. Its fibrillogenesis in biological milieu is predominantly regulated by dermatan sulfate proteoglycans, proteins conjugated with iduronic acid-containing dermatan sulfate (DS) glycosaminoglycans (GAG). Although DS is known to regulate tissue function through its modulation of Coll I architecture, a precise understanding of the latter remains elusive. We investigated this problem by visualizing the fibrillar pattern of fixed Coll I gels polymerized in the presence of varying concentrations of DS using second harmonic generation microscopy. Measuring mean second harmonic generation signal (which estimates the ordering of the fibrils), and surface occupancy (which estimates the space occupied by fibrils) supported by confocal reflectance microscopy, our observations indicated that the effect on fibril pattern of DS is contextual upon the latter's concentrations: Lower levels of DS resulted in sparse disorganized fibrils; higher levels restore organization, with fibrils occupying greater space. An appropriate change in elasticity as a result of DS levels was also observed through atomic force microscopy. Examination of dye-based GAG staining and scanning electron microscopy suggested distinct constitutions of Coll I gels when polymerized with higher and lower levels of DS. We observed that adhesion of the invasive ovarian cancer cells SKOV3 decreased for lower DS levels but was partially restored at higher DS levels. Our study shows how the Coll I gel pattern-tuning of DS is of relevance for understanding its biomaterial applications and possibly, pathophysiological functions.
\end{abstract}

\section{KEYWORDS}

collagen I, dermatan sulfate, second harmonic generation
Abbreviations: AFM, atomic force microscopy; Coll I, Collagen I or Type 1 collagen; DS, dermatan sulfate; ECM, extracellular matrix; GAG, glycosaminoglycans; IdoA, iduronic acid; PG, proteoglycans; SEM, scanning electron microscopy; SHG, second harmonic generation; SLRP, small leucine-rich proteoglycans.

Jyothsna K M and Purba Sarkar contributed equally to this study.

All authors have seen and approved the final version of the manuscript being submitted. They warrant that the article is the authors' original work, has not received prior publication and is not under consideration for publication elsewhere.

\section{1 | INTRODUCTION}

Collagen is the most abundant constituent of extracellular matrix (ECM), which is an essential component of the microenvironment of a functional tissue. ${ }^{1}$ Apart from contributing to tissue function, it provides structural integrity and mechanical durability to physiologically functional tissues and organs. ${ }^{2,3}$ Within the family of collagens, the 
most prolifically expressed Type 1 Collagen or Collagen I (Coll I) polymerizes into fibrillar scaffolds in which cells of tissues are embedded. ${ }^{1,4}$ The fibril architecture of Coll I can be modified by sulfated proteoglycans, which are proteins with long iterative chains of sulfated disaccharides known as glycosaminoglycans (GAGs). The expression and secretion of proteoglycans are tissue-specific; therefore proteoglycans contribute to making ECM distinct and specific to the tissues that secrete and remodel them. ${ }^{5,6}$ The largest class of proteoglycans comprises small leucine-rich proteoglycans (SLRPs). ${ }^{7}$ SLRPs are known to regulate collagen fibrillogenesis. ${ }^{8,9}$ They modulate collagen growth and organization of fibrils and fibers, ECM assembly, and by extension cell-matrix interactions, which are quintessential for cellular and tissue homeostasis. ${ }^{10}$ GAGs have for long been used also in the studies involving the use of biomaterials and implants which often use hydrogels, including but not limited to Coll I, where their ability to modulate hydrogel architecture and their biocompatibility are of immense benefit. ${ }^{11-13}$

Proteoglycans containing GAGs known as dermatan sulfates (DS) are differentially expressed during developmental, physiological and pathological contexts. ${ }^{7}$ DS consists of iterative units of $\mathrm{N}$-acetylgalactosamine and iduronic acid (IdoA). ${ }^{14,15}$ DS is the most abundant of GAGs that is, associated with Coll I and has been proposed to act by changing the geometry of the latter within stromal spaces. ${ }^{16}$ Reese et al polymerized Coll I in the presence of DS and DSPGs. Using scanning electron microscopy (SEM) as well as confocal reflectance microscopy, they found that DS increases the diameter of collagen fibrils. ${ }^{9}$ On the other hand, Douglas et al have observed a thinning of Coll I and II fibrils upon addition of GAGs including DS. ${ }^{17}$ The contradiction in the literature adds to the need of a precise quantitative demonstration of how and whether DS acts as a tuner of collagen gel architecture. This demonstration is crucial to the elucidation of mechanisms underlying the collagen related etiopathology of several diseases. Classic examples include congenital disorders, such as the Ehler Danlos syndrome, spondyloepimetaphyseal dysplasia, San Filippo disease, and several cancers where the DS or its conjugated protein (such as decorin, and biglycan), are upregulated. ${ }^{18-22}$ Contemporary advances in microscopic modalities can provide fresh insights into physiological and pathological dynamics of fibrillar ECM topologies.

One such modality, second harmonic generation imaging is a well-established nonlinear optical contrast mechanism for imaging biological samples. ${ }^{23-25}$ Demonstrated in biological samples first in 1986 , it is based on the upconversion of two lower energy photons to twice the incident frequency. ${ }^{26}$ Second harmonic signal is observed from objects that lack a center of symmetry, such as fibrillar ECM proteins and cellular cytoskeleton. ${ }^{27}$ The associated chirality and noncentrosymmetry of collagen along with its highly ordered arrangement (tropocollagen assembling into fibrils, which in turn assemble into fibers) makes it an exceptionally strong source of SHG. ${ }^{28,29}$ Unlike fluorescence microscopy, the strong SHG signal from collagen allows label-free imaging of tissue samples without causing phototoxicity or photobleaching. ${ }^{30}$ Moreover, it can be used to image structures in their native state in contrast with electron microscopic methods, which require sample dehydration or freezing. Such non-native state imaging can show significantly altered sample organization when compared to its native state. Since it is a nonlinear optical process involving two excitation photons, its axial and lateral resolution is higher (typically few $100 \mathrm{~nm}$ ) when compared to diffraction limited resolution of single excitation photon. ${ }^{24}$

Any attempt to get quantitative information about the biological specimen using SHG microscopy, or any optical imaging technique in general has to address the heterogeneity in the different fields-ofview, the dependence on the choice of the focal plane, the inherent optical resolution limited by the diffraction limited point-spread function of the imaging technique and its impact on the quantitative measurement. In the context of SHG microscopy, the nonlinear signal generation mechanism leads to quadratic scaling of the SHG signal with the number of nonlinear dipole oscillators. Notwithstanding these limitations, SHG microscopy has been successfully applied in the past as a potential tool for analyzing changes in the collagen morphology in many diseased states. ${ }^{28}$ SHG images combined with image analysis tools have been used to glean useful quantitative information, such as fibril width, contrast, correlation, energy, and homogeneity using image transforms such as curvelet transform, Fourier Transform matrix and grey-level co-occurrence matrix, which were successful in elucidating structural changes accompanying ovarian cancer development. $^{31,32}$ Above all, polarization resolved SHG metrices, such as collagen peptide pitch angles, SHG signal anisotropy, SHG circular dichroism, have also been used to classify ovarian cancer tissues at different stages. ${ }^{33}$

In this manuscript, using SHG imaging we have studied the effect of DS on the gel architecture of Coll I in detail. An integrative analysis of specific imaging parameters supported by confocal reflectance-, scanning electron-, and atomic force- microscopy provides insights into the alterations of element patterns by DS in a concentrationdependent and surprisingly, nonlinear fashion. Our experiments represent an approach-and-assay "toolkit" that reveals how DS alters Coll I architecture and can be extended to investigations on the regulation of fibrillar ECM topologies in general.

\section{2 | METHODS}

\subsection{Sample preparation}

Acid extracted rat tail Type I Collagen ( $3 \mathrm{mg} / \mathrm{ml}$; Thermo Fisher) was maintained at $4^{\circ} \mathrm{C}$ until polymerization. Dermatan sulfate sodium salt from porcine intestinal mucosa (Sigma-Aldrich) was reconstituted at $5 \mathrm{mg} / \mathrm{ml}$. For collagen scaffolds, Coll I was neutralized in high salt conditions ( $\times 10$ DMEM) using $2 \mathrm{~N} \mathrm{NaOH}$. Appropriate volumes of DS GAGs were then added to neutralized collagen such that the final ratio of collagen: DS was 40:1, 20:1, 5:1, 2.5:1, and 2:1 referred to as DS $(25 \mu \mathrm{g} / \mathrm{ml}), \mathrm{DS}(50 \mu \mathrm{g} / \mathrm{ml}), \mathrm{DS}(200 \mu \mathrm{g} / \mathrm{ml}), \mathrm{DS}(400 \mu \mathrm{g} / \mathrm{ml})$, and DS $(500 \mu \mathrm{g} / \mathrm{ml})$. Phosphate buffered saline (PBS) was used to adjust the final volume. The mixtures were then plated onto chambered slides and incubated for $24 \mathrm{hr}$ at $37^{\circ} \mathrm{C}$ in a carbon dioxide incubator for in vitro polymerization (See Figure 1a). At the end of $24 \mathrm{hr}$, the 
(a)

\begin{tabular}{|c|c|c|c|c|c|c|c|}
\hline Coll I & $\begin{array}{c}\text { Neutralized } \\
\text { Coll I }\end{array}$ & $\begin{array}{l}\text { Control } \\
\text { Coll }\end{array}$ & $\begin{array}{l}\text { DS:Coll } \\
(1: 40)\end{array}$ & $\begin{array}{l}\text { DS:Coll } \\
(1: 20)\end{array}$ & $\begin{array}{l}\text { Ds:Coll } \\
(1: 5)\end{array}$ & $\begin{array}{l}\text { DS:Coll } \\
\text { (1:2.5) }\end{array}$ & $\begin{array}{l}\text { DS:Coll } \\
(1: 2)\end{array}$ \\
\hline$+10 \mathrm{X}$ DMEM & Q & $\begin{aligned} \text { Coll I } \\
1 \mathrm{mg} / \mathrm{ml}\end{aligned}$ & $\begin{array}{c}\text { Coll I } \\
1 \mathrm{mg} / \mathrm{ml}\end{array}$ & $\begin{array}{c}\text { Coll I } \\
1 \mathrm{mg} / \mathrm{ml}\end{array}$ & $\underset{\substack{\text { Coll I } \\
1 \mathrm{mg} / \mathrm{ml}}}{-}$ & $\underset{1 \mathrm{mg} / \mathrm{ml}}{\text { Coll I }}$ & $\begin{array}{l}\text { Coll I } \\
1 \mathrm{mg} / \mathrm{ml}\end{array}$ \\
\hline+ PBS & & . & $\begin{array}{c}\text { DS } \\
25 \mu \mathrm{\mu g} / \mathrm{ml}\end{array}$ & $\begin{array}{c}\mathrm{Ds} \\
\mathbf{5 0} \mathrm{\mu g} / \mathrm{ml}\end{array}$ & 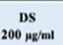 & $\begin{array}{c}\text { DS } \\
400 \mathrm{\mu y} / \mathrm{ml}\end{array}$ & 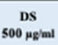 \\
\hline
\end{tabular}

(b)

$\downarrow$
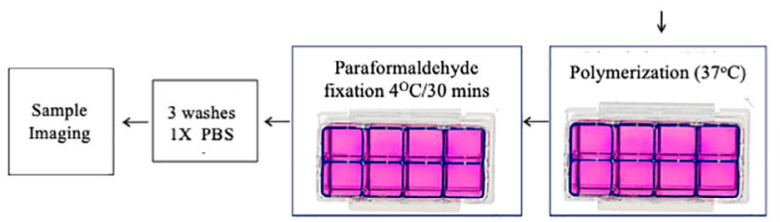

(c)
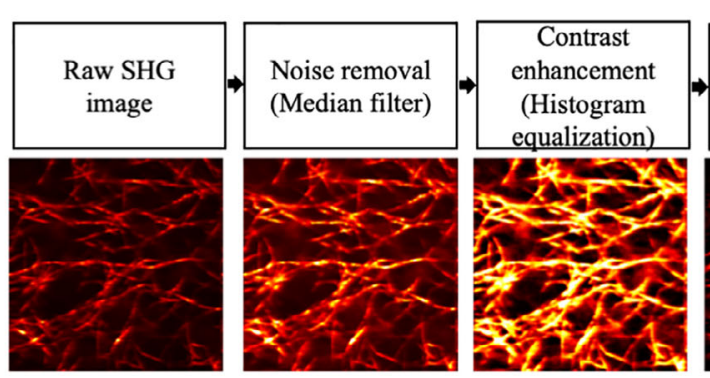

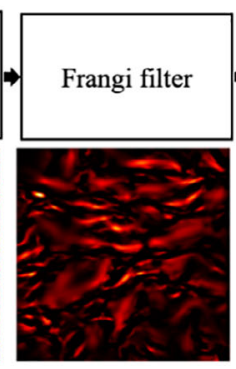

Fiber Laser (1040 nm)

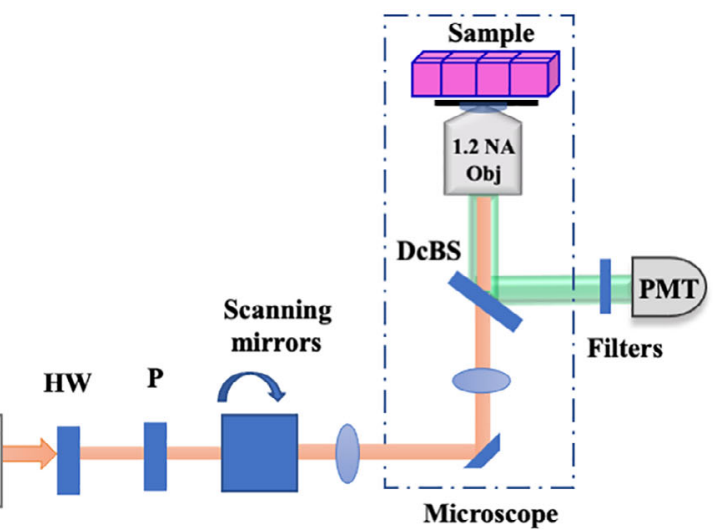

Microscope

FIGURE 1 Description of sample preparation, imaging and analysis (a) Flow chart depicting the in vitro polymerization of Coll I in the absence and presence of Dermatan Sulfate (DS) followed by fixation and preparation for imaging studies (b) Schematic depiction of the second harmonic generation (SHG) microscopic set up used for sample imaging. HW, half wave plate; PMT, photomultiplier tube; DcBS, dichroic beam splitter; $\mathrm{P}$, polarizer; and Obj, objective (c) Flow chart showing the steps involved in fibril extraction using image processing approaches

samples were fixed with $4 \%$ paraformaldehyde at $4^{\circ} \mathrm{C}$ for $30 \mathrm{~min}$, following which they were washed with PBS thrice. The fixed sample was then taken for imaging using SHG and confocal microscopy.

\subsection{Second harmonic generation and confocal microscopy}

A nonlinear microscopy set up shown in the Figure $1 \mathrm{~b}$ is used for SHG imaging of the collagen polymerized in the presence and absence of DS. An optical source of fundamental excitation at $1,040 \mathrm{~nm}$ by a femtosecond fiber laser (Fidelity HP) with $140 \mathrm{fs}$ pulse width and $80 \mathrm{MHz}$ repetition rate is used as the incident light source at the fundamental frequency. Horizontally polarized fundamental excitation is focused onto the uniformly coated collagen gels in the chamber well mounted on an Olympus IX73 inverted microscope using a $\times 60 / 1.2$ NA water immersion objective. The transverse and axial optical resolution at the SHG emission wavelength is estimated to be $\sim 470$ and $\sim 860 \mathrm{~nm}$, respectively. The second harmonic signal at $520 \mathrm{~nm}$ emitted from the sample is collected in epi-detection set up using the same objective. A dichroic mirror is used in the backward path to separate the fundamental and the second harmonic signal. The SHG signal is detected using a photomultiplier tube (Hamamatsu R3896) with a set of band-pass ( $520 \pm 20 \mathrm{~nm}$ ) and short-pass ( $890 \mathrm{~nm}$ cut-off) filters in front to reject the fundamental excitation by $\sim 200 \mathrm{~dB}$. SHG images of the collagen samples are obtained by scanning the laser beam using a pair of galvanometric mirrors (Thorlabs GVSO02). More than 15 different sample locations or field of views (FOVs) of size $50 \times 50 \mu \mathrm{m}^{2}$ are imaged from each sample by moving the motorized sample stage (Thorlab MLS203-1). The pixel resolution used in the imaging is $200 \mathrm{~nm}$. We restrict the image acquisition to a single, best focused image plane at every FOV. We acquired few images using a quarter wave-plate in the incident light path to create circularly polarized fundamental excitation and compared this with the linearly polarized excitation. The use of circularly polarized incident light did not result in any significant difference to the acquired SHG images. The epi-detected SHG signal ensures better quality imaging of the smaller fibrillar structures when compared to forward SHG signal due to the increased phase-mismatch in the backward propagation geometry for the SHG signal. ${ }^{34}$

For confocal microscopy, the collagen gels were polymerized and fixed on chambered glass slides as described in the sample preparation section. They were kept moist with PBS and imaged using $a \times 63$ oil immersion lens at the wavelength $633 \mathrm{~nm}$. A total of 15 slices (FOVs) were imaged per stack using a $0.513 \mu \mathrm{m}$ step size at a resolution of $1,024 \times 1,024$ pixels. All the confocal microscopy was performed using an inverted Zeiss LSM 880 with Airy scan, with a pixel size limited resolution of $210 \mathrm{~nm}$.

\section{3 | Image analysis}

The acquired images are processed in MATLAB and ImageJ to extract only the collagen fibrils from the images and used to calculate parameters, such as mean SHG signal, and surface occupancy. The flowchart 
showing the various image processing steps used to identify the fibrillar structures in the Collagen images is shown in Figure 1c. Median filter is used to remove the noise and adaptive histogram equalization filter is used to enhance the contrast of the raw image. Frangi filter ${ }^{35,36}$ is applied onto the contrast enhanced image to sharpen the edges of the fibrils. Binarization is done on the contrast enhanced image with and without Frangi filter using Otsu's method. Otsu's method is a nonparametric method that automates the optimal threshold calculation by using zeroth and first order cumulative moments of the gray-level histogram from the image. ${ }^{37}$ The resulting binary images are combined using OR operation to retrieve all the fibril pixels (used to calculate surface occupancy), which is further multiplied with the raw image (used to calculate mean SHG) or the contrast enhanced image to calculate the mean SHG signal. Mean SHG signal, which is a measure of the ordering of the structural elements of collagen across the imaged FOV is calculated from the above processed image by calculating the average SHG value after selecting only the image pixels corresponding to the collagen fibrils. Surface occupancy is defined as the ratio of the number of pixels occupied by the fibrils to the total number of pixels in the image. This is calculated from the binarized image by taking the ratio of number of white pixels to the total number of pixels in the binary image.

\subsection{Cell adhesion assay}

Wells of a 96-well plate were coated with appropriate matrix, depending on the experimental requirements. The matrix was polymerized for $24 \mathrm{hr}$ and was used as a substrate for adhesion of SKOV3 ovarian cancer cells, which had been cultured in McCoy's $5 \mathrm{~A}$ medium supplemented with $10 \%$ FBS. Appropriate volume of cell suspension $(10,000$ cells) was added to the wells to allow adhesion. Excess nonadhered cells were washed off after the experimental time point. The cells that adhered were fixed with $3.7 \%$ paraformaldehyde, for $30 \mathrm{~min}$, followed by two washes with PBS. The cells were then stained with propidium iodide for $10 \mathrm{~min}$, followed by two washes with PBS. Fluorescence reading were taken at excitation/ emission centered at 535/617 nm wavelengths.

\subsection{Scanning electron microscopy}

SEM was performed after coating a thin layer of gold on fixed and dehydrated collagen samples on Zeiss Ultra 55 SEM Mono CL scanning electron microscope. The imaging was done with an accelerating voltage (EHT) of $5 \mathrm{kV}$ keeping the aperture at $30 \mu \mathrm{m}$.

\section{6 | Dimethylmethylene blue assay}

Sulfated GAGs may be measured directly by the use of a metachromatic dye, 1,9-Dimethylmethylene blue (DMMB, 341088, Sigma, NY). The color of the dye solution (blue) is visually distinct from that of the solution containing GAG-dye complex (purple) and thus is useful in detecting the presence of sulfated GAGs. The Coll I gels polymerized in the absence or presence of DS GAGs were washed with PBS once before the addition of DMMB dye onto the gels. The change in color indicated the extent to which the GAGs leach out of the polymerized gels.

\subsection{Atomic force microscopy}

Atomic force microscopy (AFM) was used to measure the stiffness of the Coll I scaffolds polymerized in the absence or presence of DS GAGs. The apparent modulus of elasticity of the gels was estimated using an Atomic Force Microscope (XE Bio from Park Systems, Suwon, South Korea). A V-shaped cantilever with a spherical silicon dioxide bead having a radius of curvature of 2,600 nm (AppNano HYDRA6V-200NG-TL; AppNano, Mountain View, CA) attached to its bottom, was used for making the measurements. The stiffness of the cantilever was measured using a thermal tuning method available with the AFM and was found to be $0.045 \mathrm{~N} / \mathrm{m}$. The relation between the deformation of the cantilever and the voltage on the photodetector was calibrated by indenting the cantilever on the petri dish. The calibration was done each time the laser position on the cantilever was adjusted. The cantilever speed was maintained at $0.8 \mathrm{~mm} / \mathrm{s}$ while approaching as well as retracting from the samples. The Young's modulus was obtained using the XEl software with the Hertzian contact model as follows. First, the approach region of the F- $d$ curve when the cantilever is not in contact with the sample was identified, followed by correction of the force in that region to zero. The F-d curve is linear and almost flat in this region. A straight line is fitted to this region and this line is subtracted from the F-d curve to correct for the baseline force. The elastic modulus and contact point are now obtained from the baseline-corrected F-d curve.

\section{8 | Statistical analysis}

All experiments were repeated at least three times and during each SHG imaging trial, we imaged more than 15 FOVs from each sample. Comparison between two groups was done using paired parametric Student's $t$ test and repeated measures (RM) one-way analysis of variance (ANOVA) with Dunnett's test for post hoc multiple comparisons to compare the data of across independently performed experiments to also account for day-wise data correlation. Data set with $p$-value less than .05 is considered statistically significant. All the analyses were done using the GraphPad Prism software. Supplementary File 1 summarizes the values of the estimated parameters and the specific statistical tests for all individual experiments.

\section{3 | RESULTS}

\subsection{Incorporation of DS alters the ordering of Coll I fibrils}

The analysis of the effect of DS on the ordering/regularity of Coll I fibril patterns was carried out by comparing mean SHG signals. Mean 
pixel intensity and hence mean SHG signal is expected to be higher, when the fibrils are aligned (and even bundled) with respect to each other. ${ }^{38}$ When imaged, the fibrils of the polymerized and fixed control Coll I gels appeared organized with parallel alignments in several fields, which is prognostic of bundling of fibrils into higher order structural elements like fibers (Supplementary Figure 1A). In comparison, fibrils of Coll I polymerized with low concentration of DS (henceforth abbreviated as "low DS-Coll I") $(50 \mu \mathrm{g} / \mathrm{ml})$ showed a more disorganized web-like arrangement with much lower frequency of bundling (Figure 2ai-ii,b). The mean SHG of low DS-Coll I gels measured across independent biological repeats was significantly lower than that of control Coll I gels (Figure 2a; at an even lower DS concentration, that is, $25 \mu \mathrm{g} / \mathrm{ml}$, the SHG was insignificantly altered relative to controls (Supplementary Figure 2A). To our surprise Coll I, when polymerized at a relatively higher concentration of DS (henceforth abbreviated as "high DS-Coll I") (we have used a concentration of $400 \mu \mathrm{g} / \mathrm{ml}$ based on the previous experimental literature on the use of such concentrations of DS as well other GAGs ${ }^{39-49}$; DS, copiously expressed within connective stromal tissues is further upregulated due to several fold increase in function of enzymes such as its epimerase in cancers ${ }^{50}$ ) showed fibrils that were visually indistinguishable from control Coll I and also a higher frequency of organized and often bundled appearance (Figure 2ai,iii). Correspondingly, the mean SHG of high DS-Coll I gels was closer to, and insignificantly different from control Coll I gels (Figure 2b); this was true of a DS concentration of $200 \mu \mathrm{g} / \mathrm{ml}$ as well (Supplementary Figure 2B), whereas for $500 \mu \mathrm{g} / \mathrm{ml} \mathrm{DS}$, the mean SHG was significantly higher than control Coll I gels (Supplementary Figure 2C).

\subsection{Incorporation of DS in Coll I alters the occupancy of the fibrils on the scaffold surfaces}

The second parameter we estimated was surface occupancy, which describes the packing of the collagen fibrils in the given space or volume. When imaged with SHG imaging, fibrils were found to occupy a lower proportion of the imaged plane in low DS-Collagen I gels relative to control Coll I gels (Figure 3ai-ii,b). On the other hand, the mean surface occupancy for high DS-Coll I gels was found to be insignificantly different from control Coll I gels (Figure 3ai and iii,b). Mean surface occupancy was also seen to insignificantly altered in Coll I gels polymerized with DS of concentration 25,200 , and $400 \mu \mathrm{g} / \mathrm{ml}$ when compared with control Coll I samples (Supplementary Figure 3A-C).

\section{3 | Change in pattern of Coll I fibrils by DS imaged by SHG is consistent across fields of image acquisition}

The calculation of the above-studied two imaging parameters were arrived at through acquiring and combining measurements across $>15$ fields of view of scaffolds for each gel samples within independent biological experiment. We therefore sought to confirm the veracity of the trends assimilated across the three independent experiments, by inferring them within separate single biological experiments. The order of the parameter magnitudes (mean values) was similar for within independent experiments, with the low DS-Coll I gels correlating with lower fibril ordering and surface occupancy. High DS-Coll I

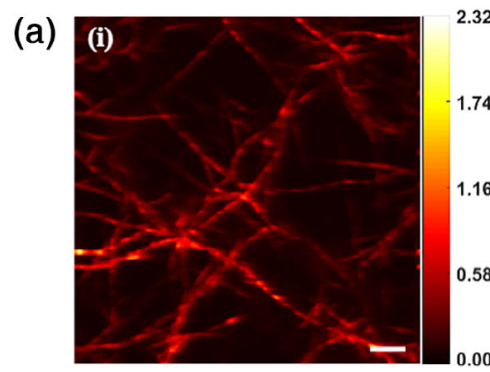

Control Coll I

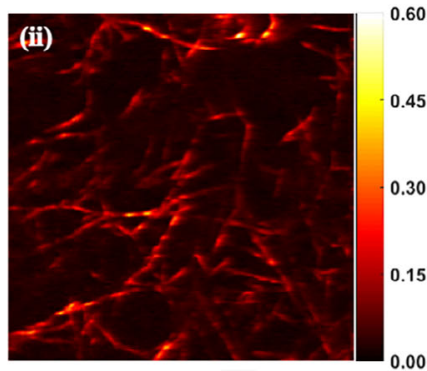

Low DS-Coll I

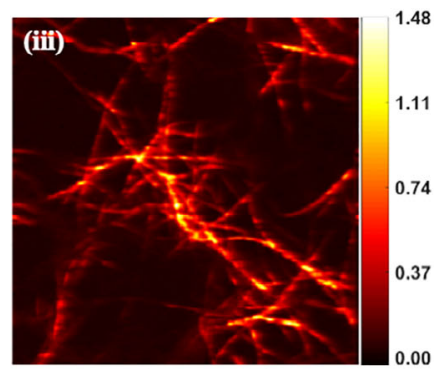

High DS-Coll I

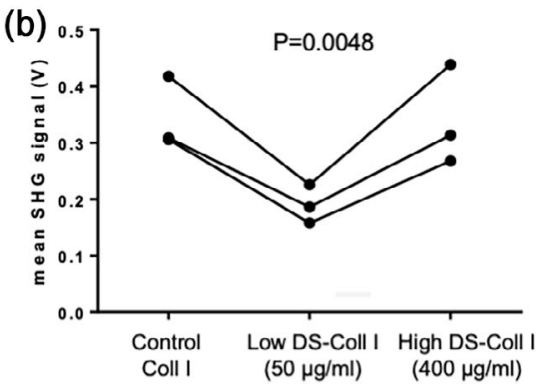

FIGURE 2 Effect of Dermatan Sulfate (DS) on mean second harmonic generation (SHG) signal of Coll I fibril architecture (a) SHG photomicrographs demonstrating the SHG signal from coll I matrices polymerized in the absence of DS (i) and presence of low DS-Coll I (ii) and high DS-Coll I (iii) respectively. Color bar labels show PMT signal in Volts and scale bar $=5 \mu \mathrm{m}$. (b) Symbol-and-line plots showing the mean SHG signal under the conditions indicated in 2A across three biological replicates. Statistical significance was computed using randomized block/ repeated measures one-way analysis of variance (ANOVA) with Dunnett's post hoc multiple comparison test 


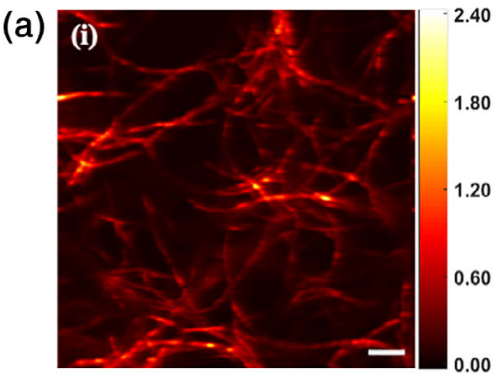

Control Coll

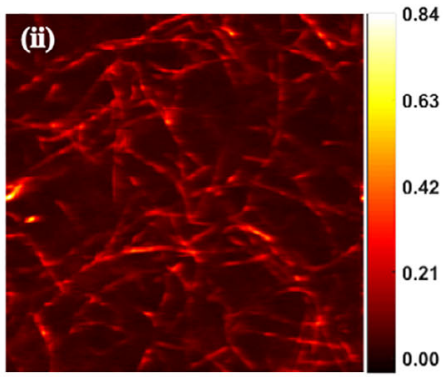

Low DS-Coll I

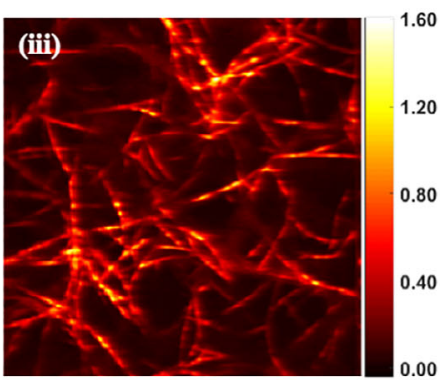

High DS-Coll I (b)

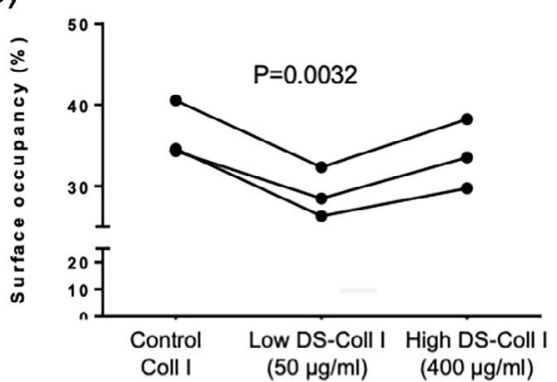

FIGURE 3 Effect of Dermatan Sulfate (DS) on mean surface occupancy of Coll I fibril architecture (a) second harmonic generation (SHG) photomicrographs demonstrating the SHG signal from Coll I matrices polymerized in the absence of DS (i) and presence of DS at $50 \mu \mathrm{g} / \mathrm{ml}$ (low DS-Coll I) (ii) and $400 \mu \mathrm{g} / \mathrm{ml}$ (high DS-Coll I) (iii) respectively. Color bar labels show PMT signal in Volts and scale bar $=5 \mu \mathrm{m}$. (b) Symbol-and-line plots showing surface occupancy under the conditions indicated in 2A across three biological replicates. Statistical significance was computed using randomized block repeated measures one-way analysis of variance (ANOVA) with Dunnett's post hoc multiple comparison test
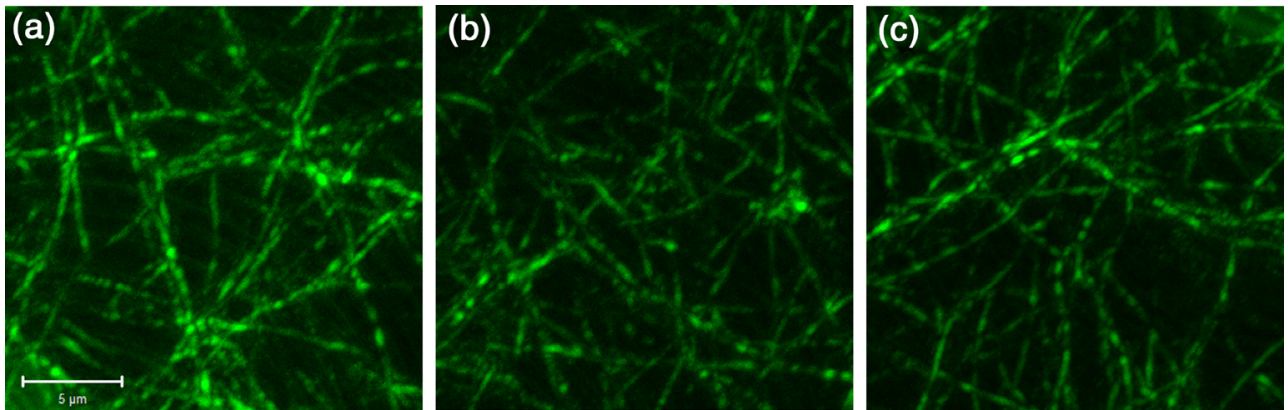

FIGURE 4 Confocal microscopic examination of Collagen I fibrils. Laser confocal reflectance micrographs of Coll I matrices polymerized in the absence of dermatan sulfate (DS) (a) and presence of $50 \mu \mathrm{g} / \mathrm{ml}$ DS (low DS-Coll I) (b) and $400 \mu \mathrm{g} / \mathrm{ml}$ DS (high DS-Coll I) (c) respectively, showing reflectance signals emanating from the collagen fibrils post polymerization and fixation. Scale bar $=5 \mu \mathrm{m}$

gels on the other hand were able to restore fibril ordering and surface occupancy to control levels. Supplementary Figure 4 (A and B) shows the differences in means of SHG and surface occupancy within a representative biological repeat.

\section{4 | Imaging of Coll I using laser confocal microscopy supports the SHG microscopy-based results}

To further confirm the results obtained from quantitative analysis done on SHG images using an independent linear optical method, we acquired confocal images of the reflectance signals emanated by control Coll I, low- and high- DS-Coll I gels. Figure 4a-c shows the representative confocal images of control Coll I, low DS-Coll I $(50 \mu \mathrm{g} / \mathrm{ml})$ and high DS-Coll I $(400 \mu \mathrm{g} / \mathrm{ml})$ respectively. For the same laser intensity, the reflectance signals from control Coll I and high DS-Coll I scaffolds were higher than that for low DS-Coll I counterparts, which showed lower signals. Numerous long and parallel fibrils could also be appreciated in the control Coll I and high DS-Coll I gels (see also Supplementary Figure 1B). In low DS-Coll I gels, shorter fibrils could be visualized at sparser densities. However, the high DS-Coll I $(400 \mu \mathrm{g} / \mathrm{ml})$ scaffold showed a dense network of longer fibrils similar to control. Taken in conjunction with our SHG imaging results, these observations allow us to suggest that the presence of lower levels of DS results in a lower tendency to bundle and form higher order 
FIGURE 5 Scanning electron microscope (SEM) images of distinct Coll I gel architectures. SEM micrographs of Coll I matrices polymerized in the absence of dermatan sulfate (DS) (a) and presence of DS $50 \mu \mathrm{g} / \mathrm{ml}$ DS (low DS-Coll I) (b) and $400 \mu \mathrm{g} / \mathrm{ml} \mathrm{DS}$ (high DS-Coll I) (c), respectively. Inset of (c) shown at higher resolution in (d). Yellow dotted lines denote long bundled Coll I fibers seen in control and high DS-Coll I gels. Blue dotted lines denote presence of non-fibrillar aggregative patches seen only in high DS-Coll I gels
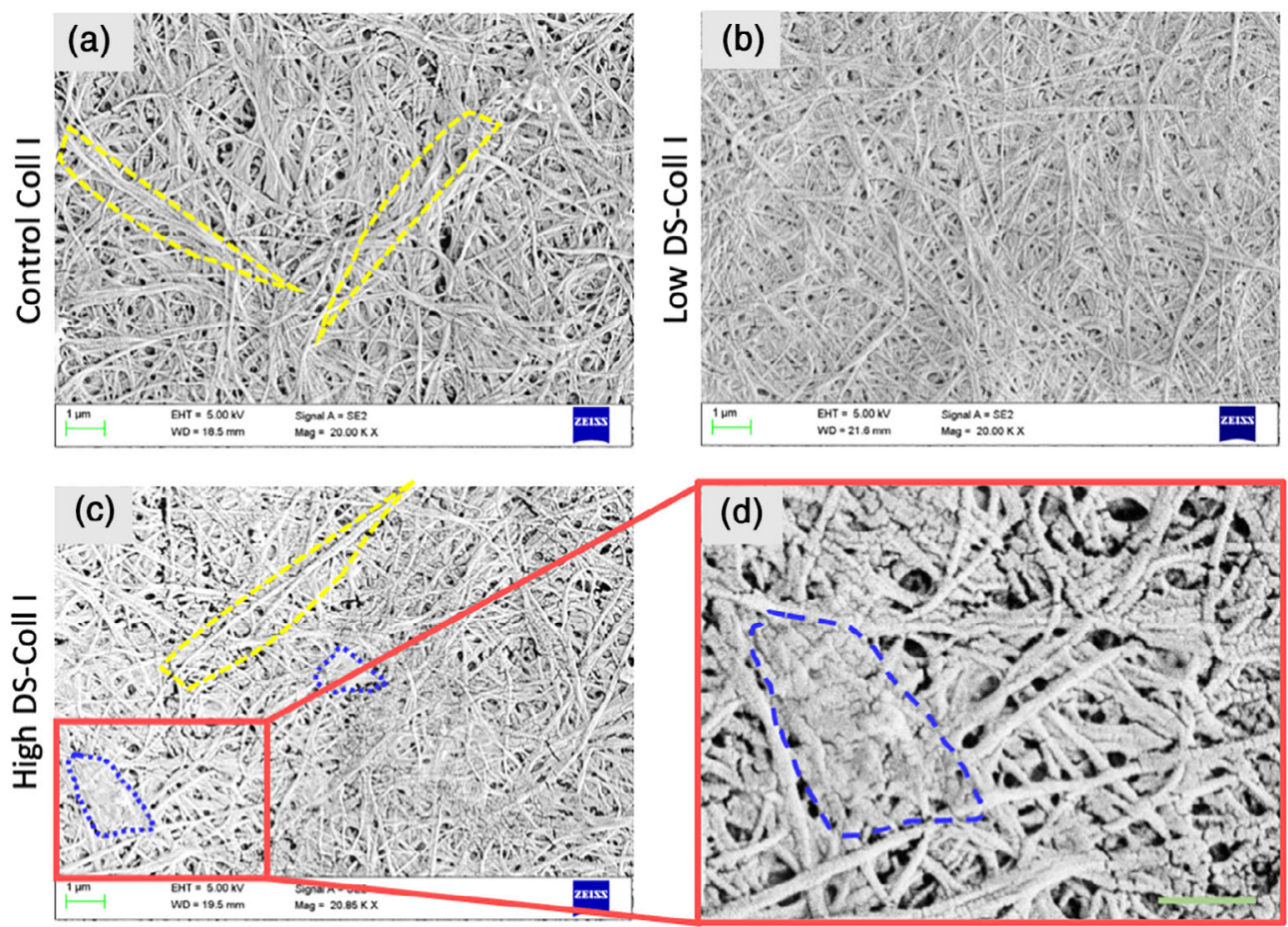

elements leading to poorer surface occupancy. Presence of higher levels of DS restores ordering and packing of fibrils.

Signatures of bundling of individual Coll I fibrils (within yellow dotted lines) into more organized structures were also observed using SEM within dehydrated and fixed gels of Coll I and high DS-Coll I, but not in low DS-Coll I scaffolds (Figure 5). In addition, we observed numerous spatial zones within high DS-Coll I gels, which were devoid of fibrillar elements (within blue dotted lines). This was neither observed in control Coll I nor in low DS-Coll I gels.

Dermatan sulfates have been observed to show self-aggregation. ${ }^{51,52}$ Therefore, we hypothesized that higher concentrations of DS self-associated, permitting Coll I to polymerize similar to pure control Coll I gels, hence the presence of non-fibrillar patches interspersed within Coll I fibrils. On the other hand, lower levels of DS dispersed better and altered the polymerization of Coll I fibrils. We examined the hypothesis by staining the gels with DMMB, a dye that is, specific to sulfated GAGs (and in this case, DS). High DS-Coll I gels were purple after polymerization and requisite washes. In contrast, control Coll I- and low DS-Coll I gels stained blue suggesting absence of substantial levels of DS, suggesting that washing low DS-Coll I gels leached out the hydrophilic DS, although the fibrillar architecture of Coll I was changed by its presence during polymerization (Figure 6a).

\section{5 | Low DS-Coll I gels are stiffer than high DS- Coll I gels}

We asked whether the alteration of Collagen fibrillar architecture by the presence of DS during its polymerization affected the mechanical properties of the former. To test this, we assayed the elastic modulus of the gels described above by measuring the elastic modulus using atomic force microscopy. Control Coll I gels recorded a mean modulus of $3 \mathrm{kPa}$ (Figure $6(\mathrm{~b}))$. The mean elastic modulus of high DS-Coll I gels was insignificantly different from control gels. In contrast low DS-Coll I gels showed an almost two-fold increase in their elastic modulus, suggesting that the altered collagen architecture as evidenced through multiple microscopic modalities also altered the mechanical properties of the collagen scaffold.

\subsection{Adhesion of SKOV3 ovarian cancer cells on Collagen I scaffolds correlates with extent of fibril order}

We investigated if the effect of DS on Coll I fibrillar pattern leads to alteration in the properties of cells cultured on top of such scaffolds. We chose SKOV3 ovarian cancer cells since mesothelial cells of the peritoneum (a common site for ovarian cancer adhesion and colonization) are known to secrete DSPGs like decorin, which may alter the stromal Collagen architecture. ${ }^{53}$ We found that the adhesion of cells incubated on top of low DS-Coll I scaffolds was lower than controls (Figure 7; cells were stained for DNA (using DAPI) and F-actin (using phalloidin)). In contrast, the adhesion on high DS-Coll I scaffold was not significantly different from control, although the trends indicated in each repeat, it was mildly higher than adhesion on low DS-Coll I gels.

\section{4 | DISCUSSION}

In 2018, an interdisciplinary team of clinicians and scientists sought to posit the interstitium of tissues as a potential new organ. ${ }^{54}$ The 
(a)

(a) Control Coll I

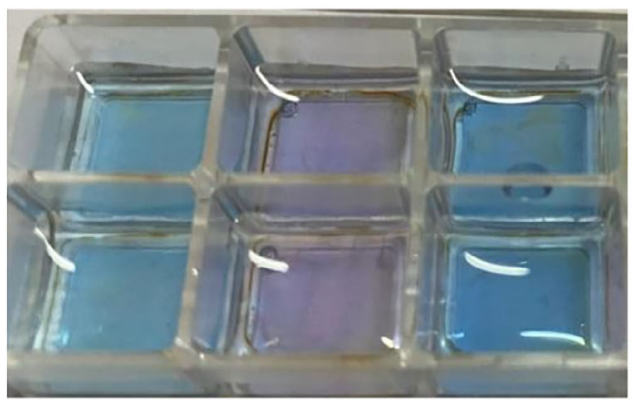

DS-Coll I

Low

DS-Coll I
High

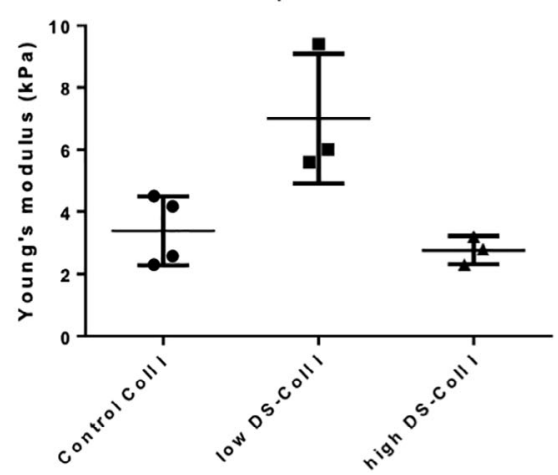

FIGURE 6 High and low dermatan sulfate (DS)-Coll I architectures have distinct compositions and elastic moduli (a) Representative gels of control Coll I (left), high DS-Coll I and low DS-Coll I (two replicates top and bottom rows) stained with DMMB (b) Measurement of Young's modulus in control Coll I, low DS- Coll I and high DS-Coll I gels as measured using atomic force microscopy. Statistical significance was computed using one-way analysis of variance (ANOVA) with Dunnett's post hoc multiple comparison test

(a)

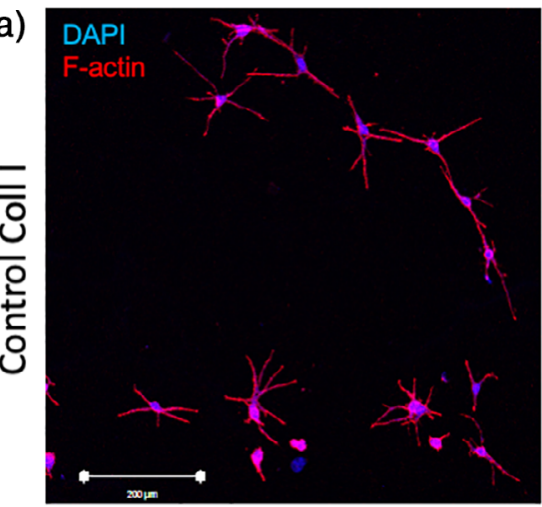

(c)

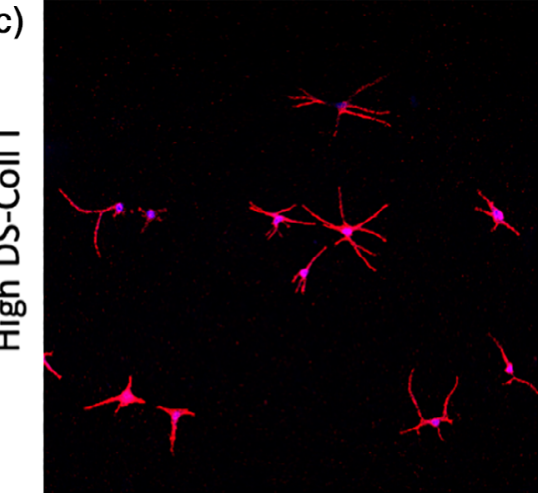

(b)
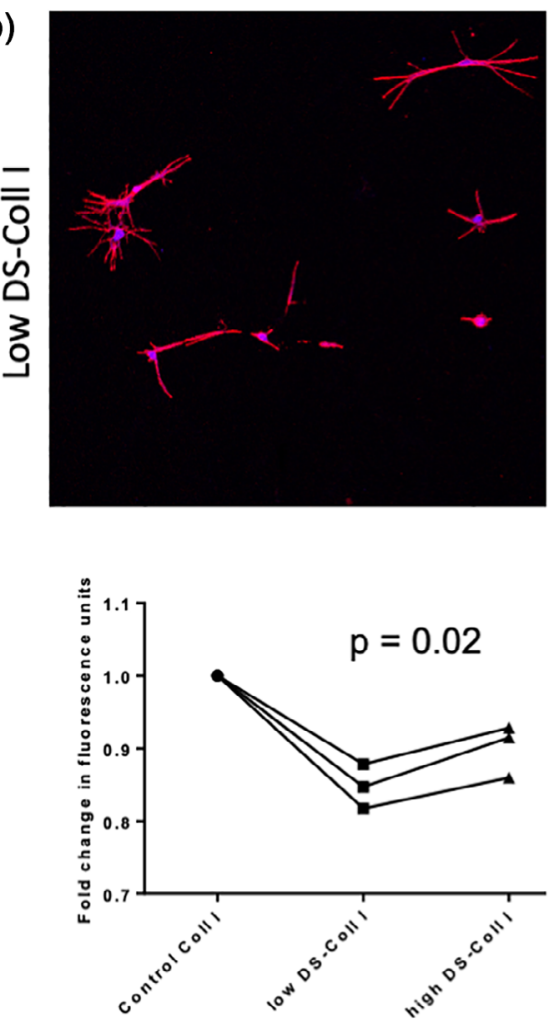

FIGURE 7 Adhesion of SKOV-3 ovarian cancer cells correlates with fibril ordering. Symbol-and-line plots showing relative adhesion of SKOV-3 ovarian cancer cells on to control Coll I (a) low dermatan sulfate (DS)-Coll I (b) and high DS-Coll I (c) respectively in three independent biological repeats. Statistical significance was computed using randomized block/repeated measures oneway analysis of variance (ANOVA) with Dunnett's post hoc multiple comparison test. Scale bar $=200 \mu \mathrm{m}$ proposition was based on the fact that histologically unprocessed organs and tissues consisted of interstitial spaces sculpted within the extracellular milieu containing ECM. Such interstitial spaces contribute to subtle functions that would otherwise not be detected when such tissues are fixed and dehydrated, resulting in loss of such spaces. The paper provided rich insights into the structure and function of interstitia using, among other microscopic techniques, SHG imaging. In fact, compressive forces acting on tissues during dehydration and sample processing may irrevocably alter the tissue microenvironment and distort the ECM architecture. This has necessitated the innovation of technologies that seek to image biological specimen in their native state as much as possible. In addition, SHG imaging has the advantage of being used to detect fibrillar structures such as Coll I in a mixture of heterogeneous biological materials.

Here, we show that SHG imaging can be used not only to detect Coll I, but is also sensitive enough to detect subtle changes in its fibril 
arrangements that are wrought by the presence of different concentrations of DS, a GAG that associates with it. In addition, our experiments help establish the regulation of Coll I fibrillogenesis by DS to be a fairly complex process: The presence of high and low levels of DS during polymerization have distinct effects on Coll I gel architecture. Whereas lower DS levels are associated with disorderly patterned fibrils, higher DS levels lead to an orderly packed fibril architecture resembling that of control Coll I scaffolds. Why may this be the case? A careful reading of the literature on DS reveals that they have a propensity for self-aggregation. ${ }^{51,55}$ An increase in levels of DS beyond a certain threshold might cause self-aggregation leading to a phase separation-like behavior, leaving Coll I fibrils to polymerize unaffected from DS. Aggregative patches in our SEM micrographs where Coll I fibrils are not present, can be visualized only in high DS samples as opposed to lower DS levels in support of such a hypothesis. These findings are further supported by the restoration of both elastic modulus and fibril pattern in these samples to control Coll I-like levels. The ratio of DS to collagen levels we have used in this study correspond with tissue- and age-specific concentrations, although the changes of such ratios in pathological conditions remains to be elucidated. ${ }^{9,56}$ Our findings may therefore also shed light on how collagen fibril architectures can diverge so drastically between, the cornea and the tendon, when the expression of DSPGs, especially decorin is thought to be ubiquitous. ${ }^{57,58}$

Our observations on DS-regulated Coll I patterns are likely to be of value for synthesis of implant scaffolds, where different greater and weaker ordered architectures can now be tuned to customize a biocompatible scaffold that provides mechanical durability and/or acts as a depot to store diffusible molecules. Furthermore, gels with loosely packed, more disordered Coll I fibers (as a result of low concentrations of DS addition) are tuned to be more elastic. The implications of our findings in the field of glycopathology are intriguing. As we show here, lower levels of DS lead to a decreased adhesion of SKOV3 ovarian cancer cells, whereas higher levels of DS seem to partially revert this effect. This may indicate that the better packed ordered fibrils on matrix-rich surfaces support better adhesion of single cancer epithelia allowing for a more efficient colonization of the niche. DSPGs, such as biglycans and decorin, are known to be upregulated in several cancers and may therefore participate in creating more habitable microenvironments by their effect on collagen fibrillar architectures. SHG imaging has been applied to investigate the morphological difference in normal and cancerous ex vivo human tissues. ${ }^{59}$ A recent study also reports the use of SHG metrices such as emission directionality, relative conversion efficiency as well as wavelet transforms and texture analysis to demonstrate the effect of decorin in organization of prostatic collagen matrix. ${ }^{60}$ Image transforms of SHG micrographs such as curvelet transform and wavelet transforms have been used to identify tumor cell boundary required for Tumor-associated collagen signature to characterize the disease progression in breast cancer models. ${ }^{61}$ Our study shows that the regulation of interstitial Coll I architecture by such DSPGs may be much more complex than previously thought with higher and lower levels of DS influencing cancer cell motility and migration in divergent ways.
We note that the SHG imaging-estimated physical parameter, such as surface occupancy, depend on the diffraction limited optical resolution of the SHG microscope $(\sim 470 \mathrm{~nm}$ for the set-up used in this work). Further improvement in optical resolution with lower wavelength excitation can help in resolving finer fibrillar features. The use of Otsu thresholding technique to clearly identify the collagen fibrillar features also inherently rejects fibrils with weak SHG signal and any out-of-focus blur from the contrast enhanced images. The use of more advanced thresholding techniques, such as multithresholding ${ }^{62}$ in recombination with feature-based selection ${ }^{63,64}$ can help minimize the rejection of collagen fibrillar units. Nonetheless, it is emphasized that the same image analysis procedure is consistently followed across the multiple concentration samples and trial runs in this study to extract statistically significant differences. The benefits of using SHG imaging makes such a study lot simpler and applicable directly to in vitro samples, even in the presence of cells without the need for complex sample preparation as required for electron microscopy.

Future efforts will be directed at understanding the role of diverse DS levels, as well as mutant DS moieties (evidenced in congenital diseases such as mucopolysachharidoses) on the manifestation and heterogeneity of cellular phenotype within collagenous microenvironments. Simultaneous forward and backward SHG imaging combined with a ratiometric approach can be applied to determine subtle changes in the collagen fibrillar pattern. ${ }^{34,65}$ Furthermore, SHG imaging can be combined with bond-specific vibrationally resonant sumfrequency generation spectroscopy ${ }^{66}$ to understand the biochemical origins of modification of the asymmetric molecular units in collagen triple helix in the presence of proteoglycans. We would also study in detail how DS-modulated divergent Coll I element architectures may exhibit distinct mechanical properties as has been elegantly shown albeit at a single DS concentration before. ${ }^{9}$ This will further the potential for DS as a tuner of the architectural properties of the most abundant ECM protein of our body with wide implications for its future use in the field of bioengineering.

\section{ACKNOWLEDGMENTS}

Ramray Bhat and Varun Raghunathan would like to acknowledge the Institute of Eminence grant (IE/CARE-19-0319) received from the Indian Institute of Science Bangalore for funding this research. This work was supported by the Wellcome Trust/DBT India Alliance Fellowship/Grant [grant number IA/I/17/2/ 503312] awarded to Ramray Bhat. Ramray Bhat would also like to acknowledge support from the Department of Biotechnology, India [BT/PR26526/GET/119/92/2017] and SERB [ECR/2015/000280]. Varun Raghunathan would also like to acknowledge support from Department of Science \& Technology through the Indo-Korea joint research projects (INT/Korea/P-44). Purba Sarkar would like to acknowledge funding support from DST INSPIRE fellowship (IF170197, IVR number- 201600020331).

\section{CONFLICT OF INTEREST}

The authors declare no potential conflict of interest. 


\section{AUTHOR CONTRIBUTIONS}

Jyothsna K M, Purba Sarkar, Keshav Kumar Jha, Varun Raghunathan and Ramray Bhat, designed the experiments. Jyothsna K M, Purba Sarkar and Keshav Kumar Jha performed the experiments. Lal Krishna A. S. performed SEM imaging. Jyothsna K M, Purba Sarkar, Keshav Kumar Jha, Varun Raghunathan and Ramray Bhat analyzed the results and wrote the manuscript.

\section{DATA AVAILABILITY STATEMENT}

The data that support the findings of this study are available from the corresponding author upon reasonable request.

\section{ORCID}

Ramray Bhat (D) https://orcid.org/0000-0002-5215-9721

\section{REFERENCES}

1. Mouw JK, Ou G, Weaver VM. Extracellular matrix assembly: a multiscale deconstruction. Nat Rev Mol Cell Biol. 2014;15(12):771-785.

2. Theocharis AD, Skandalis SS, Gialeli C, Karamanos NK. Extracellular matrix structure. Adv Drug Deliv Rev. 2016;97:4-27.

3. Bonnans C, Chou J, Werb Z. Remodelling the extracellular matrix in development and disease. Nat Rev Mol Cell Biol. 2014;15(12): 786-801.

4. Scott JE. Proteoglycan-fibrillar collagen interactions. Biochem J. 1988; 252(2):313-323.

5. Yanagishita M. Function of proteoglycans in the extracellular matrix. Acta Pathol Jpn. 1993;43(6):283-293.

6. Nash MA, Deavers MT, Freedman RS. The expression of decorin in human ovarian tumors. Clin Cancer Res. 2002;8(6):1754-1760.

7. lozzo RV, Murdoch AD. Proteoglycans of the extracellular environment: clues from the gene and protein side offer novel perspectives in molecular diversity and function. FASEB J. 1996;10(5):598-614.

8. Chen S, Birk DE. The regulatory roles of small leucine-rich proteoglycans in extracellular matrix assembly. FEBS J. 2013;280(10):21202137.

9. Reese SP, Underwood CJ, Weiss JA. Effects of decorin proteoglycan on fibrillogenesis, ultrastructure, and mechanics of type I collagen gels. Matrix Biol. 2013;32(7-8):414-423.

10. Ameye L, Young MF. Mice deficient in small leucine-rich proteoglycans: novel in vivo models for osteoporosis, osteoarthritis, Ehlers-Danlos syndrome, muscular dystrophy, and corneal diseases. Glycobiology. 2002; 12(9):107R-116R

11. Kowitsch A, Zhou G, Groth T. Medical application of glycosaminoglycans: a review. J Tissue Eng Regen Med. 2018;12(1):e23-e41.

12. Tierney CM, Jaasma MJ, O'Brien FJ. Osteoblast activity on collagenGAG scaffolds is affected by collagen and GAG concentrations. J Biomed Mater Res A. 2009;91(1):92-101.

13. Prestwich GD. Hyaluronic acid-based clinical biomaterials derived for cell and molecule delivery in regenerative medicine. J Control Release. 2011;155(2):193-199.

14. Vallen MJ, Schmidt S, Oosterhof A, Bulten J, Massuger LF, van Kuppevelt TH. Primary ovarian carcinomas and abdominal metastasis contain 4,6-disulfated chondroitin sulfate rich regions, which provide adhesive properties to tumour cells. PloS One. 2014;9(11): e111806.

15. Li HP, Komuta Y, Kimura-Kuroda J, van Kuppevelt TH, Kawano $H$. Roles of chondroitin sulfate and dermatan sulfate in the formation of a lesion scar and axonal regeneration after traumatic injury of the mouse brain. J Neurotrauma. 2013;30(5):413-425.

16. Junqueira LC, Montes GS. Biology of collagen-proteoglycan interaction. Arch Histol Jpn. 1983;46(5):589-629.
17. Douglas $\mathrm{T}$, Heinemann S, Mietrach $\mathrm{C}$, et al. Interactions of collagen types I and II with chondroitin sulfates A-C and their effect on osteoblast adhesion. Biomacromolecules. 2007;8(4):1085-1092.

18. Gouignard N, Maccarana M, Strate I, von Stedingk K, Malmstrom A Pera EM. Musculocontractural Ehlers-Danlos syndrome and neurocristopathies: dermatan sulfate is required for Xenopus neural crest cells to migrate and adhere to fibronectin. Dis Model Mech. 2016;9(6):607-620.

19. Mizumoto S, Kosho T, Yamada S, Sugahara K. Pathophysiological significance of dermatan sulfate proteoglycans revealed by human genetic disorders. Pharmaceuticals (Basel). 2017;10(2):34.

20. Nakamura A, Osonoi T, Terauchi Y. Relationship between urinary sodium excretion and pioglitazone-induced edema. J Diabetes Investig. 2010;1(5):208-211.

21. Appunni S, Anand V, Khandelwal M, Gupta N, Rubens M, Sharma A Small leucine rich proteoglycans (decorin, biglycan and lumican) in cancer. Clin Chim Acta. 2019;491:1-7.

22. Bostrom $P$, Sainio A, Eigeliene $N$, et al. Human metaplastic breast carcinoma and Decorin. Cancer Microenviron. 2017;10(1-3):39-48.

23. Pavone FS, Campagnola PJ. Second Harmonic Generation Imaging. Boca Raton, FL: CRC Press; 2016.

24. Masters BR, So P: Handbook of Biomedical Nonlinear Optical Microscopy, USA: Oxford University Press; 2008

25. Campagnola P, Dong CY. Second harmonic generation microscopy: principles and applications to disease diagnosis. Laser Photonics Rev. 2011;5(1):13-26

26. Freund I, Deutsch M, Sprecher A. Connective tissue polarity: optical second-harmonic microscopy, crossed-beam summation,and smallangle scattering in rat-tail tendon. Biophys J. 1986;50(4):693-712.

27. Campagnola PJ, Dong CY. Second harmonic generation microscopy: principles and applications to disease diagnosis. Laser Photonics Rev. 2011;5(1):13-26

28. Campagnola P. Second harmonic generation imaging microscopy: applications to diseases diagnostics. Anal Chem. 2011;83(9):32243231.

29. Rocha-Mendoza I, Yankelevich DR, Wang M, et al. Sum frequency vibrational spectroscopy: the molecular origins of the optical secondorder nonlinearity of collagen. 2007;93(12):4433-4444.

30. W M, CW F, S L, XS X. Coherent nonlinear optical imaging: beyond fluorescence microscopy. Annu Rev Phys Chem. 2011;62:507-530.

31. Campbell KR, Campagnola PJ. Assessing local stromal alterations in human ovarian cancer subtypes via second harmonic generation microscopy and analysis. J Biomed Opt. 2017;22(11):1-7.

32. Watson JM, Rice PF, Marion SL, et al. Analysis of second-harmonicgeneration microscopy in a mouse model of ovarian carcinoma. J Biomed Opt. 2012;17(7):076002.

33. Campbell KR, Chaudhary R, Handel JM, Patankar MS, Campagnola PJ. Polarization-resolved second harmonic generation imaging of human ovarian cancer. J Biomed Opt. 2018;23(6):1-8.

34. Chu S-W, Tai S-P, Chan M-C, et al. Thickness dependence of optical second harmonic generation in collagen fibrils. Opt Express. 2007;15 (19):12005-12010.

35. A F, W N, K V, M. V: Medical image computing and computerassisted Interventation. 1998.

36. Xu S, Kang CH, Gou X, et al. Quantification of liver fibrosis via second harmonic imaging of the Glisson's capsule from liver surface. J Biophotonics. 2016;9(4):351-363.

37. OTSU N. A Threshold selection method from gray-level histograms IEEE Trans Syst Man Cybern Syst. 1979;9(1):62-66.

38. Chen X, Nadiarynkh O, Plotnikov S, Campagnola PJ. Second harmonic generation microscopy for quantitative analysis of collagen fibrillar structure. Nat Protoc. 2012;7(4):654-669.

39. Muir IF, Padilla-Lamb A, Stewart JE, Wheatley DN. Growth inhibition of cultured fibroblasts by extracts from human dermis. Br J Plast Surg. 1997;50(3):186-193 
40. Kuiper NJ, Sharma A. A detailed quantitative outcome measure of glycosaminoglycans in human articular cartilage for cell therapy and tissue engineering strategies. Osteoarthr Cartil. 2015;23(12):22332241.

41. Tovar AM, de Mattos DA, Stelling MP, Sarcinelli-Luz BS, Nazareth RA, Mourao PA. Dermatan sulfate is the predominant antithrombotic glycosaminoglycan in vessel walls: implications for a possible physiological function of heparin cofactor II. Biochim Biophys Acta. 2005;1740(1):45-53.

42. Ellis BJ, Lujan TJ, Dalton MS, Weiss JA. Medial collateral ligament insertion site and contact forces in the ACL-deficient knee. J Orthop Res. 2006;24(4):800-810.

43. Lin $\mathrm{KH}$, Hino $\mathrm{H}$, Maeda S, Inagaki H, Airat JV, Saito T. Albumin synthesis by rat hepatocytes cultured on collagen gels is sustained specifically by heparin. Exp Cell Res. 1995;219(2):717-721.

44. Kano K, Miyano T, Kato S. Effects of glycosaminoglycans on the development of in vitro-matured and -fertilized porcine oocytes to the blastocyst stage in vitro. Biol Reprod. 1998;58(5):1226-1232.

45. Abbadini M, Zhu GJ, Maggi A, Pangrazzi J, Donati MB, Mussoni L. Dermatan sulphate induces plasminogen activator release in the perfused rat hindquarters. Blood. 1987;70(6):1858-1860.

46. Eriksen GV, Malmstrom A, Uldbjerg N, Huszar G. A follicular fluid chondroitin sulfate proteoglycan improves the retention of motility and velocity of human spermatozoa. Fertil Steril. 1994;62(3): 618-623.

47. Lin $\mathrm{KH}$, Maeda S, Inagaki H, Saito T. Long-term culture of primary rat hepatocytes on heparin- or lambda carrageenan-containing collagen gels. Biosci Biotechnol Biochem. 1997;61(6):971-974.

48. Obrink B. A study of the interactions between monomeric tropocollagen and glycosaminoglycans. Eur J Biochem. 1973;33(2):387-400.

49. McLaughlin RW, De Stigter JK, Sikkink LA, Baden EM, RamirezAlvarado M. The effects of sodium sulfate, glycosaminoglycans, and Congo red on the structure, stability, and amyloid formation of an immunoglobulin light-chain protein. Protein Sci. 2006;15(7):17101722.

50. Thelin MA, Svensson KJ, Shi X, et al. Dermatan sulfate is involved in the tumorigenic properties of esophagus squamous cell carcinoma. Cancer Res. 2012;72(8):1943-1952.

51. Coster L, Fransson LA, Sheehan J, Nieduszynski IA, Phelps CF. Selfassociation of dermatan sulphate proteoglycans from bovine sclera. Biochem J. 1981;197(2):483-490.

52. Racey TJ, Rochon P, Mori F, Neville GA. Examination of a possible role for dermatan sulfate in the aggregation of commercial heparin samples. J Pharm Sci. 1989;78(3):214-218.

53. Yung S, Thomas GJ, Stylianou E, Williams JD, Coles GA, Davies M. Source of peritoneal proteoglycans. Human peritoneal mesothelial cells synthesize and secrete mainly small dermatan sulfate proteoglycans. Am J Pathol. 1995;146(2):520-529.

54. Benias PC, Wells RG, Sackey-Aboagye B, et al. Structure and distribution of an unrecognized Interstitium in human tissues. Sci Rep. 2018;8 (1):4947.
55. Scott PG, Nakano T, Dodd CM, Pringle GA, Kuc IM. Proteoglycans of the articular disc of the bovine temporomandibular joint. II. Low molecular weight dermatan sulphate proteoglycan. Matrix. 1989;9(4): 284-292.

56. McKee TJ, Perlman G, Morris M, Komarova SV. Extracellular matrix composition of connective tissues: a systematic review and metaanalysis. Sci Rep. 2019;9(1):10542.

57. Mauviel A, Santra M, Chen YQ, Uitto J, lozzo RV. Transcriptional regulation of decorin gene expression. Induction by quiescence and repression by tumor necrosis factor-alpha. J Biol Chem. 1995;270(19):11692-11700.

58. Trowbridge JM, Gallo RL. Dermatan sulfate: new functions from an old glycosaminoglycan. Glycobiology. 2002;12(9):117R-125R.

59. Chen X, Huang Z, Xi G, Chen Y, Lin D, Wang J, Li Z, Sun L, Chen J, Chen R: Quantitative analysis of collagen change between normal and cancerous thyroid tissues based on SHG method. In: Tenth international conference on photonics and imaging in biology and medicine (PIBM 2011). 2012.

60. Campbell KR, Chaudhary R, Montano M, lozzo RV, Bushman WA, Campagnola PJ. Second-harmonic generation microscopy analysis reveals proteoglycan decorin is necessary for proper collagen organization in prostate. J Biomed Opt. 2019;24(6):1-8.

61. Tilbury K, Campagnola PJ. Applications of second-harmonic generation imaging microscopy in ovarian and breast cancer. Perspect Medicin Chem. 2015;7:21-32.

62. LIAO P-S, CHEN T-S, CHUNG P-C. A fast algorithm for multilevel thresholding. J Inf Sci Eng. 2001;17(5):713-727.

63. Bredfeldt JS, Liu Y, Pehlke CA, et al. Computational segmentation of collagen fibers from second-harmonic generation images of breast cancer. J Biomed Opt. 2014;19:016007.

64. LBM G, Alex C-T, Wang F, et al. Collagen morphology and texture analysis: from statistics to classification. Sci Rep. 2013;3(2190):1-10. https://doi.org/10.1038/srep02190.

65. Williams RM, Zipfel WR, Webb WW. Interpreting second-harmonic generation images of collagen I fibrils. Biophys J. 2005;88:1377-1386.

66. Raghunathan V, Han Y, Korth O, Ge NH, Potma EO. Rapid vibrational imaging with sum frequency generation microscopy. Opt Lett. 2011; 36(19):3891-3893.

\section{SUPPORTING INFORMATION}

Additional supporting information may be found online in the Supporting Information section at the end of this article.

How to cite this article: Jyothsna KM, Sarkar $P$, Jha KK, $A$. S. LK, Raghunathan V, Bhat R. A biphasic response of polymerized Type 1 collagen architectures to dermatan sulfate. J Biomed Mater Res. 2021;1-11. https://doi.org/10. 1002/jbm.a.37160 